\title{
Free Subspaces of Free Locally Convex Spaces
}

\author{
Saak S. Gabriyelyan ${ }^{1}$ and Sidney A. Morris $\mathbb{D D}^{2,3}$ \\ ${ }^{1}$ Department of Mathematics, Ben-Gurion University of the Negev, P.O. 653, Beer-Sheva, Israel \\ ${ }^{2}$ Faculty of Science and Technology, Federation University Australia, P.O. Box 663, Ballarat, VIC 3353, Australia \\ ${ }^{3}$ Department of Mathematics and Statistics, La Trobe University, Melbourne, VIC 3086, Australia
}

Correspondence should be addressed to Sidney A. Morris; morris.sidney@gmail.com

Received 24 September 2017; Accepted 10 December 2017; Published 24 January 2018

Academic Editor: Hugo Leiva

Copyright (c) 2018 Saak S. Gabriyelyan and Sidney A. Morris. This is an open access article distributed under the Creative Commons Attribution License, which permits unrestricted use, distribution, and reproduction in any medium, provided the original work is properly cited.

If $X$ and $Y$ are Tychonoff spaces, let $L(X)$ and $L(Y)$ be the free locally convex space over $X$ and $Y$, respectively. For general $X$ and $Y$, the question of whether $L(X)$ can be embedded as a topological vector subspace of $L(Y)$ is difficult. The best results in

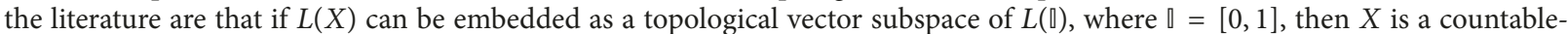
dimensional compact metrizable space. Further, if $X$ is a finite-dimensional compact metrizable space, then $L(X)$ can be embedded as a topological vector subspace of $L(\mathbb{\square})$. In this paper, it is proved that $L(X)$ can be embedded in $L(\mathbb{R})$ as a topological vector subspace if $X$ is a disjoint union of a countable number of finite-dimensional locally compact separable metrizable spaces. This is the case if $X=\mathbb{R}^{n}, n \in \mathbb{N}$. It is also shown that if $\mathbb{G}$ and $Q$ denote the Cantor space and the Hilbert cube $\mathbb{\mathbb { N }}^{\mathbb{N}}$, respectively, then (i) $L(X)$ is embedded in $L(\mathbb{G})$ if and only if $X$ is a zero-dimensional metrizable compact space; (ii) $L(X)$ is embedded in $L(Q)$ if and only if $Y$ is a metrizable compact space.

\section{Introduction}

For a Tychonoff space $X$, we denote by $L(X), \mathbb{V}(X), F(X)$, and $A(X)$ the free locally convex space, the free topological vector space, the free topological group, and the free abelian topological group over $X$, respectively. These spaces and groups always exist and are essentially unique; see [1-5].

Let $X$ and $Y$ be Tychonoff spaces. The questions of whether $L(X)$ and $\mathbb{V}(X)$ can be embedded as a topological vector space in $L(Y)$ and $\mathbb{V}(Y)$, respectively, and whether $F(X)$ and $A(X)$ can be embedded as a topological group in $F(Y)$ and $A(Y)$, respectively, have been studied for many years; see for example [6-8]. The case $Y=\mathbb{\square}:=[0,1]$ was studied in [7] where the following result is obtained.

Theorem 1 (see [7]). If $K$ is a finite-dimensional metric compact space, then $L(K)$ is embedded in $L(\mathbb{\square})$ as a topological vector space.

The proof of this theorem in [7] is nontrivial and uses the deep and powerful Kolmogorov Superposition Theorem which answered Hilbert's 13th Problem. In the last section of this paper, we give a simpler application of the Kolmogorov Superposition Theorem to obtain Theorem 1.

In this paper we answer similar questions for when $\square$ is replaced with the Cantor space $\mathbb{G}$ or the Hilbert cube $Q=\mathbb{\mathbb { N }}^{\mathbb{N}}$. We determine what Tychonoff spaces $Y$ are such that $L(Y)$ can be embedded as a topological vector subspace in $L(\mathbb{G})$ and $L(Q)$, respectively.

It is well-known that $\mathbb{G}$ is universal for all zerodimensional compact metrizable spaces and $Q$ is universal for all compact metrizable spaces. So it is straightforward to prove that if $Z$ is a compact metrizable space, then $L(Z)$ can be embedded as a topological vector subspace in $L(Q)$, and if $Z$ is a zero-dimensional compact metrizable space, then $L(Z)$ can be embedded as a topological vector subspace of $L(\mathbb{G})$. Of course both of these embeddings are the natural ones. But are there other Tychonoff spaces $Z$ such that $L(Z)$ can be embedded as a topological vector subspace of $L(Q)$ or $L(\mathbb{G})$ in a less natural way? A partial answer to this question was given in [8] where this was answered in the negative for compact spaces. 
Theorem 2 (see [8]). If $Z$ is a compact Hausdorff space, then

(i) $L(Z)$ is embedded as a topological vector subspace of $L(Q)$ if and only if $Z$ is metrizable;

(ii) $L(Z)$ is embedded as a topological vector subspace of $L(\mathbb{G})$ if and only if $Z$ is metrizable and zerodimensional.

In Theorem 11 of this paper we prove that the condition "compact" is unnecessary, more precisely, if $L(Z)$ is embedded in $L(Q)$ or $L(\mathbb{G})$, then $Z$ must to be compact.

Related questions are as follows: let $Y$ be a compact metrizable space and $X$ a subspace of $Y$. What are the conditions on $X$ under which each of the following is true: (i) $L(X)$ can be embedded as a topological vector subspace of $L(Y)$; (ii) $\mathbb{V}(X)$ can be embedded as a topological vector subspace of $\mathbb{V}(Y) ; F(X)$ can be embedded as a topological subgroup of $F(Y) ; A(X)$ can be embedded as a topological subgroup of $A(Y)$. For the special case $Y=\square$ complete answers to this question are given in $[7,9]$.

Theorem 3. For a subspace $X$ of $\square$ the following are equivalent:

(i) ([7]) $A(X)$ is embedded into $A(\square)$ as a topological subgroup;

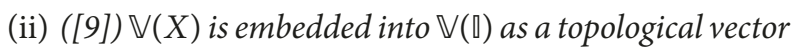
subspace;

(iii) $X$ is locally compact.

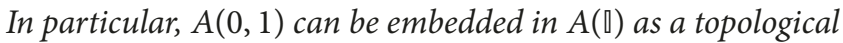
subgroup and $\mathbb{V}(0,1)$ can be embedded in $\mathbb{V}(\square)$ as a topological vector subspace.

For the case of free locally convex spaces on compact metrizable spaces $Y$, our Theorem 8 gives a complete description of those subspaces $X$ of $Y$ with the property that $L(X)$ can be embedded as a topological vector subspace of $L(Y)$. Our proof is based on significant generalizations of some results in [7].

\section{The Free Locally Convex Spaces on the Cantor Space and the Hilbert Cube}

Let us recall (see [1]) that the free locally convex space $L(X)$ on a Tychonoff space $X$ is a pair consisting of a locally convex space $L(X)$ and a continuous map $i: X \rightarrow L(X)$ such that every continuous map $f$ from $X$ to any locally convex space $E$ gives rise to a unique continuous linear operator $\bar{f}: L(X) \rightarrow$ $E$ with $f=\bar{f} \circ i$. The free locally convex space $L(X)$ always exists and is essentially unique. The set $X$ forms a Hamel basis for $L(X)$, and the map $i$ is a topological embedding $[3,10]$.

It is well-known (see [3]) that the dual space of $L(X)$ is canonically isomorphic to the space $C(X)$ of all continuous real-valued functions on $X$. The space $C(X)$ endowed with the pointwise topology or with the compact-open topology is denoted by $C_{p}(X)$ and $C_{k}(X)$, respectively. Denote by $L_{p}(X)$ the space $L(X)$ endowed with the weak topology. Then the spaces $L_{p}(X)$ and $C_{p}(X)$ are in duality.
Firstly we note the following useful necessary condition for the existence of an embedding for free locally convex spaces which easily follows from general facts of locally convex space theory.

Proposition 4. Let $X$ and $Y$ be Tychonoff spaces.

(i) If the space $L(X)$ can be embedded into $L(Y)$ as a locally convex subspace, then also the space $L_{p}(X)$ can be embedded into $L_{p}(Y)$ as a locally convex subspace.

(ii) The space $L_{p}(X)$ can be embedded into $L_{p}(Y)$ as a locally convex subspace if and only if $C_{p}(X)$ is an image of $C_{p}(Y)$ under a linear continuous surjection.

Consequently, if $L(X)$ can be embedded into $L(Y)$ as a locally convex subspace, then there is a linear continuous surjection $T$ from $C_{p}(Y)$ onto $C_{p}(X)$.

Proof. (i) follows from Theorem 8.12.2 of [11], and (ii) follows from Theorems 8.10 .5 and 8.13 .2 of [11] applying to $\mathscr{X}:=$ $C_{p}(Y)$ and $\mathcal{Y}:=C_{p}(X)$.

Note that the converse assertion in (i) of Proposition 4 holds; for example, if $L(X)$ is barrelled, see Corollary 11.3.7 of [11]. However, the condition on $L(X)$ to be a barrelled space is very restrictive: the space $L(X)$ is barrelled if and only if $X$ is discrete; see Theorem 6.4 of [4].

Let $X$ and $Y$ be Tychonoff spaces. To obtain sufficient conditions on $X$ and $Y$ for which $L(X)$ is embedded into $L(Y)$, by Proposition 4, it is sufficient to find conditions on $X$ and $Y$ under which the converse assertion in (i) of Proposition 4 holds. The case when $Y$ is compact (or, more generally, $Y$ is a $k_{\omega}$-space) is the most natural and important.

Recall that a Tychonoff space $X$ is called Dieudonné complete if its topology is induced by a complete uniformity. Every Lindelöf space (in particular, every $k_{\omega}$-space) and every metrizable space is Dieudonné complete. We shall use the following result of Arhangelskii.

Theorem 5 (see [12]). Let $X$ and $Y$ be Dieudonné complete spaces. If a linear map $h: C_{p}(X) \rightarrow C_{p}(Y)$ is continuous, then $h$ is continuous as a map $C_{k}(X) \rightarrow C_{k}(Y)$.

Recall that a map $f: X \rightarrow Y$ is called compact-covering if for every compact subset $C$ of $Y$ there is a compact subset $K$ of $X$ such that $f(K)=C$.

Proposition 6. Let $X$ be a Dieudonné complete space and let $Y$ be a $k_{\omega}$-space. If $T: C_{p}(Y) \rightarrow C_{p}(X)$ is a linear continuous surjection, then $X$ is also a $k_{\omega}$-space and $T: C_{k}(Y) \rightarrow C_{k}(X)$ is a quotient compact-covering map. If $Y$ is additionally a compact space, then $X$ is a compact space. If $Y$ is compact metrizable, then so is $X$.

Proof. Since any $k_{\omega}$-space is Dieudonné complete, the map $T$ is also continuous with respect to the compact-open topologies on both spaces by Theorem 5 . As $C_{k}(Y)$ is a Fréchet space, the Open Mapping Theorem [11, Theorem 14.4.6] implies that $T: C_{k}(Y) \rightarrow C_{k}(X)$ is open, and hence it is a quotient map. Therefore $C_{k}(X)$ is also a Fréchet space, so $X$ is 
a $k_{\omega}$-space. Since $C_{k}(Y)$ and $C_{k}(X)$ are completely metrizable and $T$ is open, the map $T$ is compact-covering by Theorem 1.2 of [13]. If, in addition, $Y$ is a (metrizable) compact space, then $C_{k}(Y)$ is a (separable) Banach space, so is $C_{k}(X)$. Thus $X$ is also a (metrizable) compact space.

The equivalence (i) $\Leftrightarrow$ (ii) in the next theorem generalizes Lemma 3.2 of [7] and has an essentially simpler proof.

Proposition 7. Let $X$ be a Dieudonné complete space and let $Y$ be a $k_{\omega}$-space (a compact space or a metrizable compact space). Then the following assertions are equivalent:

(i) $L(X)$ can be embedded into $L(Y)$ as a locally convex subspace.

(ii) $L_{p}(X)$ can be embedded into $L_{p}(Y)$ as a locally convex subspace.

(iii) There is a linear continuous surjection of $C_{p}(Y)$ onto $C_{p}(X)$.

If (i)-(iii) hold, the space $X$ is a $k_{\omega}$-space (a compact space or a metrizable compact space, resp.).

Proof. (i) $\Rightarrow$ (ii) and (ii) $\Leftrightarrow$ (iii) follow from Proposition 4 . Let us prove (ii) $\Rightarrow$ (i).

Let $h: L_{p}(X) \rightarrow L_{p}(Y)$ be an embedding of locally convex spaces. Then the dual linear map $h^{*}: C_{p}(Y) \rightarrow C_{p}(X)$ is a continuous surjection by Theorems 8.10 .5 and 8.11.3 of [11]. By Proposition $6, X$ is a $k_{\omega}$-space (a compact space or a metrizable compact space, resp.) and $h^{*}: C_{k}(Y) \rightarrow$ $C_{k}(X)$ is a quotient compact-covering map. Hence the dual continuous map $h^{* *}$ of $h^{*}$ from the space $C_{k}\left(C_{k}(X)\right)$ to $C_{k}\left(C_{k}(Y)\right)$ is an embedding of $C_{k}\left(C_{k}(X)\right)$ into $C_{k}\left(C_{k}(Y)\right)$ as a locally convex subspace. Observe that since $X$ and $Y$ are $k_{\omega}$-spaces, $L(X)$ and $L(Y)$ are locally convex subspaces of $C_{k}\left(C_{k}(X)\right)$ and $C_{k}\left(C_{k}(Y)\right)$, respectively, by $[10,14]$ (for a more general assertion, see Theorem 1.2 of [15]). This observation and the fact that $h$ coincides with the restriction of $h^{* *}$ to $L(X)$ imply that the map $h$ is an embedding of $L(X)$ into $L(Y)$.

We can now easily deduce the following main result from Proposition 7 and Lemma 3.3 of [16].

Theorem 8. Let $X$ be a subspace of a compact metrizable space $K$. Then $L(X)$ can be embedded into $L(K)$ as a locally convex subspace if and only if $X$ is closed.

Proof. Assume that $L(X)$ embeds into $L(K)$. Then $X$ is compact by Proposition 7, and so $X$ is closed. The converse assertion follows from Lemma 3.3 of [16].

It follows immediately from Theorem 8 that $L(0,1)$ is not embedded in $L(\mathbb{\square})$. This fact contrasts with the facts, mentioned in Theorem 3 , that $A(0,1)$ can be embedded as a topological subgroup of $A(\mathbb{\square})$ and $\mathbb{V}(0,1)$ can be embedded as a topological vector subspace of $\mathbb{V}(\mathbb{\square})$.

It turns out that the existence of a linear continuous surjection $T$ from $C_{p}(Y)$ onto $C_{p}(X)$ in Propositions 4 and 7 is also a sufficiently strong condition as the following easy corollary of Uspenskiı̌'s theorems [17] shows.
Theorem 9 (see [7]). Let $T: C_{p}(Y) \rightarrow C_{p}(X)$ be a linear continuous surjection. If $Y$ is a metrizable compact space, then so is $X$.

Proposition 4 and Theorem 9 immediately imply the following result.

Corollary 10. Let $X$ be a Tychonoff space and let $K$ be a compact metrizable space. If $L(X)$ can be embedded into $L(K)$ as a locally convex subspace, then $X$ is compact and metrizable.

Corollary 10 and Theorem 2 imply the following surprising complete descriptions of Tychonoff spaces $X$ for which $L(X)$ is embedded in $L(Q)$ and $L(X)$ is embedded in $L(\mathbb{G})$.

Theorem 11. For a Tychonoff space $X$ the following assertions are equivalent:

(i) $L(X)$ can be embedded into $L(Q)$ as a locally convex subspace.

(ii) There is a linear continuous surjection of $C_{p}(Q)$ onto $C_{p}(X)$.

(iii) $X$ is a compact metrizable space.

Proof. (i) $\Rightarrow$ (ii) follows from Proposition 4 , (ii) $\Rightarrow$ (iii) follows from Theorem 9 , and (iii) $\Rightarrow$ (i) follows from Theorem 2 .

Theorem 12. For a Tychonoff space $X$ the following assertions are equivalent:

(i) $L(X)$ can be embedded into $L(\mathbb{G})$ as a locally convex subspace.

(ii) There is a linear continuous surjection of $C_{p}(\mathbb{G})$ onto $C_{p}(X)$.

(iii) $X$ is a compact zero-dimensional metrizable space.

Proof. (i) $\Rightarrow$ (ii) follows from Proposition 4 . (ii) $\Rightarrow$ (i): by Theorem $9, X$ is compact and metrizable and Proposition 7 applies.

(i) $\Rightarrow$ (iii): If $L(X)$ can be embedded into $L(\mathbb{G})$, then $X$ is compact and metrizable by Corollary 10 . Therefore $X$ is zerodimensional by Theorem 2 . (iii) $\Rightarrow$ (i) follows from Theorem 2 .

Corollary 13. Let $K$ be a zero-dimensional metrizable compact space and $X$ a Tychonoff space. If $L(X)$ can be embedded into $L(K)$ as a locally convex subspace, then $X$ is a compact zero-dimensional metrizable space.

Proof. By Theorem 12, $L(K)$ is embedded into $L(\mathbb{G})$ and Theorem 12 applies once again.

Note that the locally compact pseudocompact space $\omega_{1}\left(=\left[0, \omega_{1}\right)\right)$ is not Dieudonné complete and its Dieudonné completion is the compact space $\omega_{1}+1$. By [18, Example 3.1.27]; the restriction continuous map $R: C_{p}\left(\omega_{1}+1\right) \rightarrow$ $C_{p}\left(\omega_{1}\right)$ is bijective. However $R$ is not open because the set

$$
R\left(\left\{f \in C\left(\omega_{1}+1\right):\left|f\left(\omega_{1}\right)\right| \leq 1\right\}\right)
$$

is not a neighborhood of zero in $C_{p}\left(\omega_{1}\right)$. 
Question 14. Do there exist a compact (nonmetrizable) space $K$ and a non-Dieudonné complete subspace $X$ of $K$ such that $L(X)$ cannot be embedded into $L(K)$ ? What can be said about the specific case: $K=\omega_{1}+1$ and $X=\omega_{1}$ ?

\section{Embedding into $L(X)$ and the Kolmogorov Superposition Theorem}

Now we consider an important case when a linear continuous surjection of $C_{p}(Y)$ onto $C_{p}(X)$ can be easily constructed using the following deep result of Kolmogorov [19] in the version of Lorentz [20, Theorem 11.1].

Theorem 15 (Kolmogorov superposition theorem). For every $n \in \mathbb{N}$ there exist strictly increasing functions $\varphi_{1}, \ldots, \varphi_{2 n+1} \in$

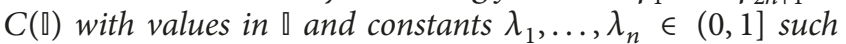
that for every function $f\left(x_{1}, \ldots, x_{n}\right) \in C\left(\square^{n}\right)$ there is a function $g \in C[0, n]$ such that

$$
f=\sum_{q=1}^{2 n+1} g\left(\sum_{i=1}^{n} \lambda_{i} \varphi_{q}\left(x_{i}\right)\right)
$$

Corollary 16. Let $K$ be a finite-dimensional compact metrizable space. Then there is a linear continuous surjective map $T$ from $C_{p}(\square)$ onto $C_{p}(K)$.

Proof. By the Embedding Theorem [21, Theorem 1.11.4], every finite-dimensional compact metrizable space $K$ is embedded into $\mathbb{R}^{n}$ for some $n \in \mathbb{N}$. Now the Tietze-Urysohn Theorem shows that to prove the corollary it is sufficient to construct a linear continuous surjective map $T$ from $C_{p}(\square)$ onto $C_{p}\left(\square^{n}\right)$.

Define the homeomorphism $h:[0, n] \rightarrow \square$ by $h(x):=x / n$. Now, using (2) we define the operator $T: C_{p}(\square) \rightarrow C_{p}\left(\square^{n}\right)$ by

$$
\begin{aligned}
& T(g)\left(x_{1}, \ldots, x_{n}\right):=\sum_{q=1}^{2 n+1} g \circ h\left(\sum_{i=1}^{n} \lambda_{i} \varphi_{q}\left(x_{i}\right)\right) \\
& g \in C(\mathbb{\square}) .
\end{aligned}
$$

It is clear that $T$ is linear, and $T$ is surjective by the Kolmogorov Theorem. To check that $T$ is continuous, fix $z_{j}=$ $\left(x_{1}^{j}, \ldots, x_{n}^{j}\right), j=1, \ldots, m$, and $\varepsilon>0$. Denote by $F$ the finite

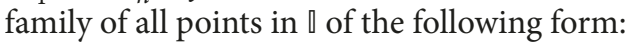

$$
h\left(\sum_{i=1}^{n} \lambda_{i} \varphi_{q}\left(x_{i}^{j}\right)\right), \quad j=1, \ldots, m, q=1, \ldots, 2 n+1 .
$$

Then, if $g$ belongs to the standard open pointwise neighborhood $\{f \in C(\mathbb{\square}):|f(x)|<\varepsilon /(2 n+1) \forall x \in F\}$ of zero function in $C_{p}(\mathbb{\square})$, we obtain

$$
\begin{aligned}
\left|T(g)\left(z_{i}\right)\right| \leq \sum_{q=1}^{2 n+1}\left|g \circ h\left(\sum_{i=1}^{n} \lambda_{i} \varphi_{q}\left(x_{i}^{j}\right)\right)\right|<\varepsilon, & \\
& j=1, \ldots, m .
\end{aligned}
$$

Thus $T$ is continuous.
Remark 17. Levin informed the authors that a linear continuous surjective map $T$ from $C_{p}(\square)$ onto $C_{p}(K)$ can even be chosen to be open; see [22].

We now observe the following proof of Theorem 1 .

Proof. Theorem 1 follows immediately from Proposition 7 and Corollary 16.

Now we consider a noncompact case, namely, when $X=$ $\mathbb{R}$. First we recall the following definition motivated by the Kolmogorov Superposition Theorem 15.

Definition 18 (see [23]). Let $X$ be a Tychonoff space. A family $\Phi \subseteq C(X)$ is said to be basic for $X$ if each $f \in C(X)$ can be written as

$f=\sum_{q=1}^{n}\left(g_{q} \circ \Phi_{q}\right)$

for some $\Phi_{1}, \ldots, \Phi_{n} \in \Phi, g_{1}, \ldots, g_{n} \in C(\mathbb{R}), n \in \mathbb{N}$.

We shall use the following noncompact version of the Kolmogorov Superposition Theorem.

Theorem 19 (see [24]). If $X$ is a finite-dimensional locally compact separable metrizable space, then $X$ has a finite basic family. result.

Analogously to Corollary 16 we easily obtain the following

Corollary 20. Let $X$ be a finite-dimensional locally compact separable metrizable space. Then there is an $n \in \mathbb{N}$ such that $C_{p}(X)$ is the image of $\left(C_{p}(\mathbb{R})\right)^{n}$ under a continuous linear surjective map $T$.

Proof. By Theorem 19 there exists a basic family $\left\{\Phi_{n}, \ldots, \Phi_{n}\right\}$ for $X$. Then the map

$$
\begin{aligned}
& T:\left(C_{p}(\mathbb{R})\right)^{n} \longrightarrow C_{p}(X), \\
& T\left(g_{1}, \ldots, g_{n}\right):=\sum_{i=1}^{n}\left(g_{i} \circ \Phi_{i}\right),
\end{aligned}
$$

is linear and surjective. Let us check that $T$ is continuous.

Fix a standard neighborhood $\{f \in C(X):|f(x)|<$ $\varepsilon \forall x \in F\}$ of zero in $C_{p}(X)$, where $F \subseteq X$ is finite and $\varepsilon>0$. Denote by $\widetilde{F}$ the finite set of all points in $\mathbb{R}$ of the form $\Phi_{i}(z)$, where $z \in F$ and $1 \leq i \leq n$. If $g_{1}, \ldots, g_{n} \in\{h \in C(\mathbb{R})$ : $|h(z)|<\varepsilon / n \forall z \in \widetilde{F}\}$, we obtain

$$
\left|T\left(g_{1}, \ldots, g_{n}\right)(z)\right|=\left|\sum_{i=1}^{n}\left(g_{i} \circ \Phi_{i}\right)(z)\right|<\varepsilon, \quad \forall z \in F .
$$

Therefore $T$ is continuous.

Proposition 21. Let $X=\bigsqcup_{n \in \mathbb{N}} X_{n}$ be a disjoint union of finitedimensional locally compact separable metrizable spaces. Then there is a continuous linear surjective map $T$ from $C_{p}(\mathbb{R})$ onto $C_{p}(X)$. 
Proof. By Corollary 20, for every $n \in \mathbb{N}$, we can choose an $s_{n} \in$ $\mathbb{N}$ and a continuous linear surjective map $T_{n}:\left(C_{p}(\mathbb{R})\right)^{s_{n}} \rightarrow$ $C_{p}\left(X_{n}\right)$. Then the map

$$
\begin{aligned}
T:\left(C_{p}(\mathbb{R})\right)^{\mathbb{N}} & =\prod_{n=1}^{\infty}\left(C_{p}(\mathbb{R})\right)^{s_{n}} \longrightarrow \prod_{n=1}^{\infty} C_{p}\left(X_{n}\right) \\
& =C_{p}(X), \\
T\left(\left(f_{n}\right)\right) & :=\left(T_{n}\left(f_{n}\right)\right),
\end{aligned}
$$

is continuous, linear, and surjective. It is known (see [25, Section 177]) that there is a linear homeomorphism $S$ from $C_{p}(\mathbb{R})$ onto $\left(C_{p}(\mathbb{R})\right)^{\mathbb{N}}$. Thus the map $T \circ S$ is as desired.

Now Propositions 7 and 21 immediately imply the main result of this section.

Theorem 22. If $X=\bigsqcup_{n \in \mathbb{N}} X_{n}$ is a disjoint union of finitedimensional locally compact separable metrizable spaces, then $L(X)$ can be embedded into $L(\mathbb{R})$ as a locally convex subspace. In particular, for every $n \in \mathbb{N}, L\left(\mathbb{R}^{n}\right)$ can be embedded into $L(\mathbb{R})$ as a locally convex subspace.

We conclude by noting that Theorem 22 implies that if $X$ is the countable-dimensional locally compact separable metrizable space $\bigsqcup_{n \in \mathbb{N}} \mathbb{R}^{n}$, then $L(X)$ can be embedded as a locally convex subspace of $L(\mathbb{R})$.

\section{Conflicts of Interest}

The authors declare that they have no conflicts of interest.

\section{Acknowledgments}

The authors thank Professors M. Levin and V. Tkachuk for their advice [22,24] and [25], respectively. The second author acknowledges the Ben Gurion University of the Negev for hospitality during which the research for this paper was undertaken.

\section{References}

[1] A. Markoff, “On free topological groups," Doklady Akademii Nauk SSSR, vol. 31, pp. 299-301, 1941.

[2] M. I. Graev, "Free topological groups," Izvestiya Akademii Nauk SSSR Seriya Matematicheskaya, vol. 12, pp. 279-324, 1948 (Russian), Topology and Topological Algebra. Translation Series 1, vol. 8, pp. 305-364, 1962.

[3] D. A. Rakov, "Free locally convex spaces for uniform spaces," Matematicheskii Sbornik, vol. 63, no. 105, pp. 582-590, 1964.

[4] S. S. Gabriyelyan and S. A. Morris, "Free topological vector spaces," Topology and its Applications, vol. 223, pp. 30-49, 2017.

[5] W. Grilliette, "Scaled-free objects II," Annals of Functional Analysis, vol. 6, no. 3, pp. 216-261, 2015.

[6] E. Katz, S. A. Morris, and P. Nickolas, "A free subgroup of the free abelian topological group on the unit interval," Bulletin of the London Mathematical Society, vol. 14, no. 5, pp. 399-402, 1982.
[7] A. Leiderman, S. A. Morris, and V. Pestov, "The free abelian topological group and the free locally convex space on the unit interval," Journal of the London Mathematical Society-Second Series, vol. 56, no. 3, pp. 529-538, 1997.

[8] S. S. Gabriyelyan and S. A. Morris, "Embedding into free topological vector spaces on compact metrizable spaces," Topology and its Applications, vol. 233, pp. 33-43, 2018.

[9] S. S. Gabriyelyan and S. A. Morris, "Embedding into the free topological vector space on I," Bulletin of the Australian Mathematical Society, vol. 97, pp. 110-118, 2018.

[10] V. V. Uspenskiî, "Free topological groups of metrizable spaces," Mathematics of the USSR - Izvestija, vol. 37, no. 3, pp. 657-680, 1991.

[11] L. Narici and E. Beckenstein, Topological vector spaces, vol. 296, CRC Press, Boca Raton, FL, USA, 2nd edition, 2011.

[12] A. V. Arhangelskii, "On linear homeomorphisms of function spaces," Soviet Mathematics-Doklady, vol. 25, pp. 852-855, 1982.

[13] A. V. Arhangel'skii, "Open and close-to-open mappings. Relations among spaces," Trudy Moskovskogo Matematicheskogo Obshchestva, vol. 15, pp. 181-223, 1966.

[14] J. Flood, Free locally convex spaces, Dissertationes Math CCXXI, PWN, Warczawa, Poland, 1984.

[15] S. Gabriyelyan, "On the Ascoli property for locally convex spaces," Topology and its Applications, vol. 230, pp. 517-530, 2017.

[16] S. S. Gabriyelyan, "Free locally convex spaces and the k-space property," Canadian Mathematical Bulletin, vol. 57, no. 4, pp. 803-809, 2014.

[17] V. V. Uspenski1̆, "A characterization of compactness in terms of uniform structure in a function space," Russian Mathematical Surveys, vol. 37, no. 4, pp. 143-144, 1982.

[18] R. Engelking, General Topology, Panstwowe Wydawnictwo Naukowe, Waszawa, Poland, 1977.

[19] A. N. Kolmogorov, "On the representation of continuous functions of many variables by superposition of continuous functions of one variable and addition," American Mathematical Society Translations, vol. 28, pp. 55-61, 1963.

[20] G. G. Lorentz, Approximation of Functions, Holt, Rinehart and Winston, Chicago, Ill, USA, 1966.

[21] R. Engelking, Dimension Theory, PWN-Polish Scientific Publishers, Warsaw, Poland, 1978.

[22] M. Levin, "A property of $C_{p}[0,1]$," Transactions of the American Mathematical Society, vol. 363, no. 5, pp. 2295-2304, 2011.

[23] Y. Sternfeld, "Hilbert's 13th problem and dimension," in Geometric Aspects of Functional Analysis, vol. 1376 of Lecture Notes in Mathematics, pp. 1-49, Springer, Berlin, Germany, 1989.

[24] Y. Hattori, "Dimension and superposition of bounded continuous functions on locally compact, separable metric spaces," Topology and its Applications, vol. 54, no. 1-3, pp. 123-132, 1993.

[25] V. V. Tkachuk, A Cp-theory Problem Book. Topological and Function Spaces, Problem Books in Mathematics, Springer, New York, NY, USA, 2011. 


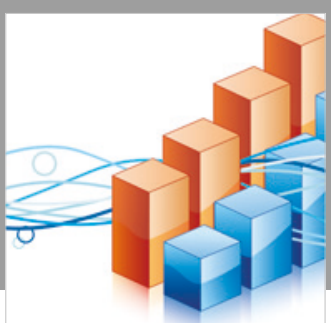

Advances in

Operations Research

\section{-n-m}
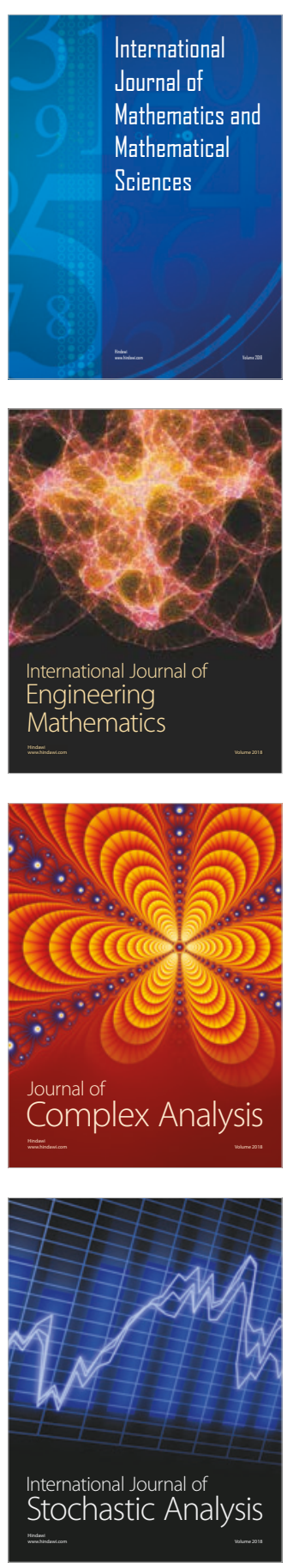
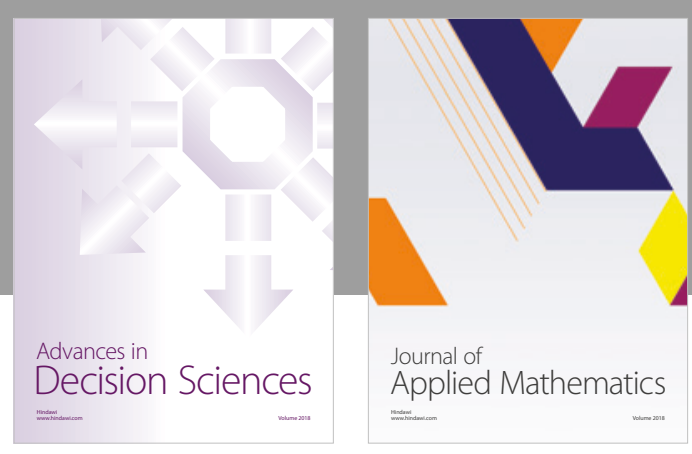

Journal of

Applied Mathematics
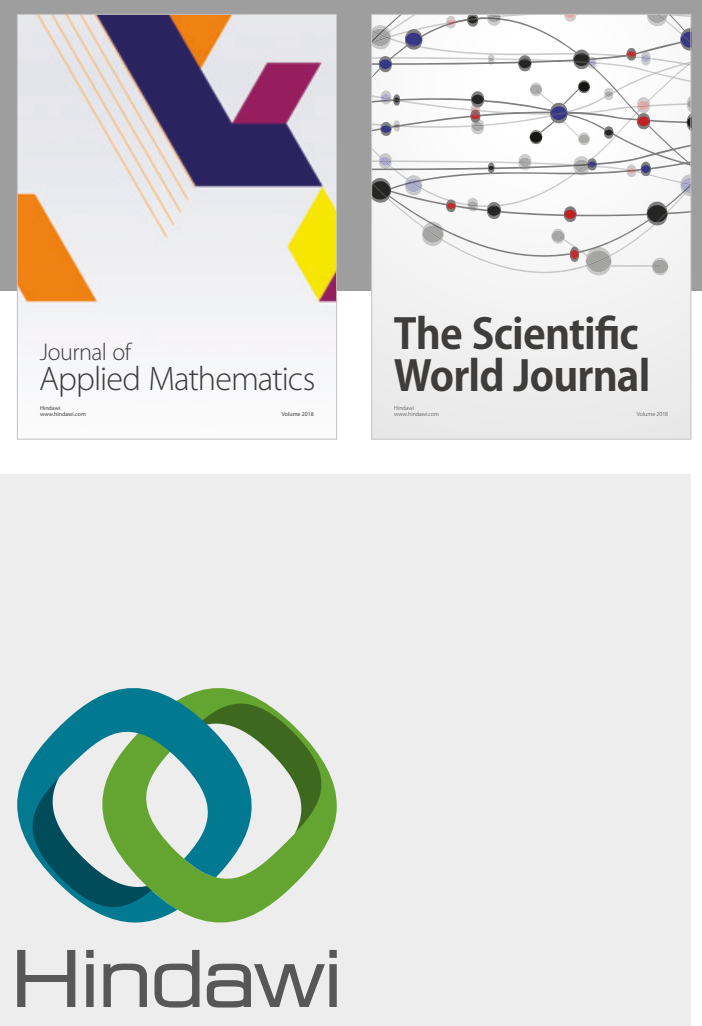

Submit your manuscripts at

www.hindawi.com

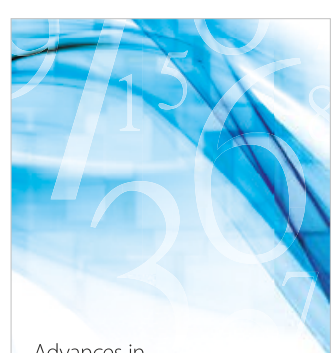

Advances in
Numerical Analysis
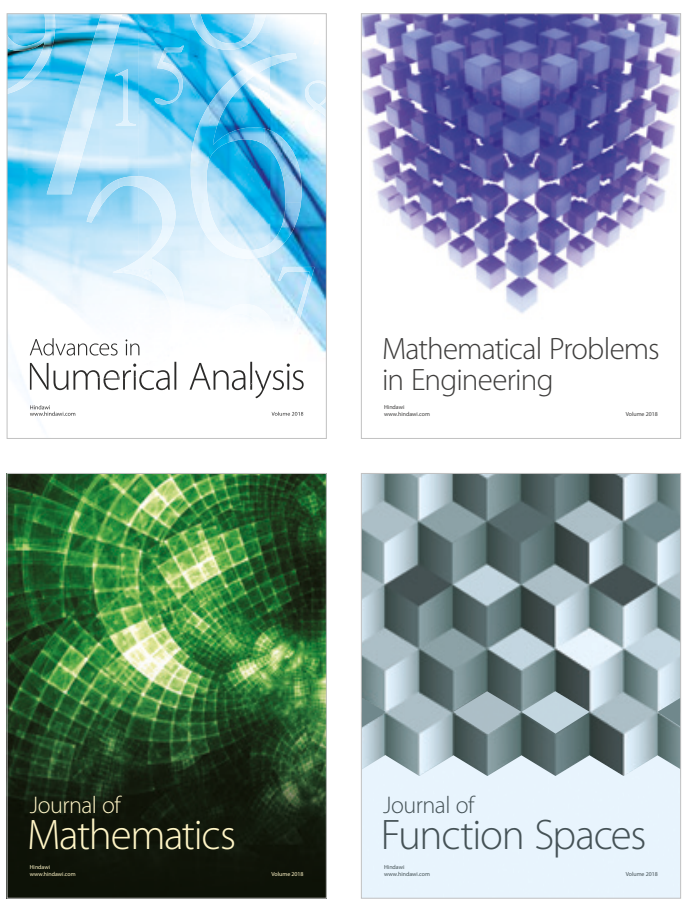

Mathematical Problems in Engineering

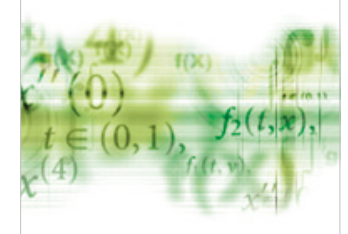

International Journal of

Differential Equations

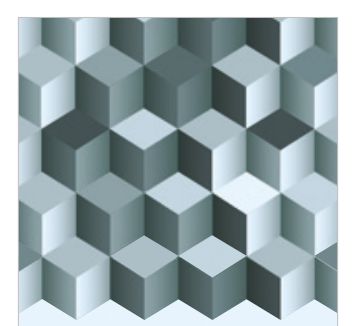

Journal of

Function Spaces

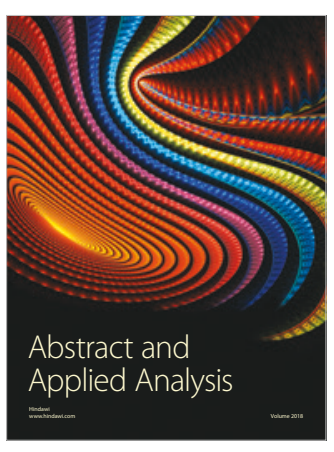

The Scientific

World Journal

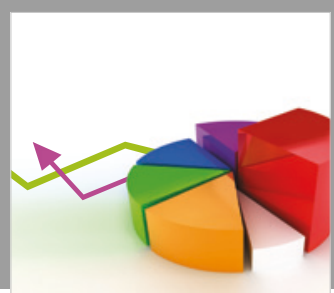

Journal of

Probability and Statistics
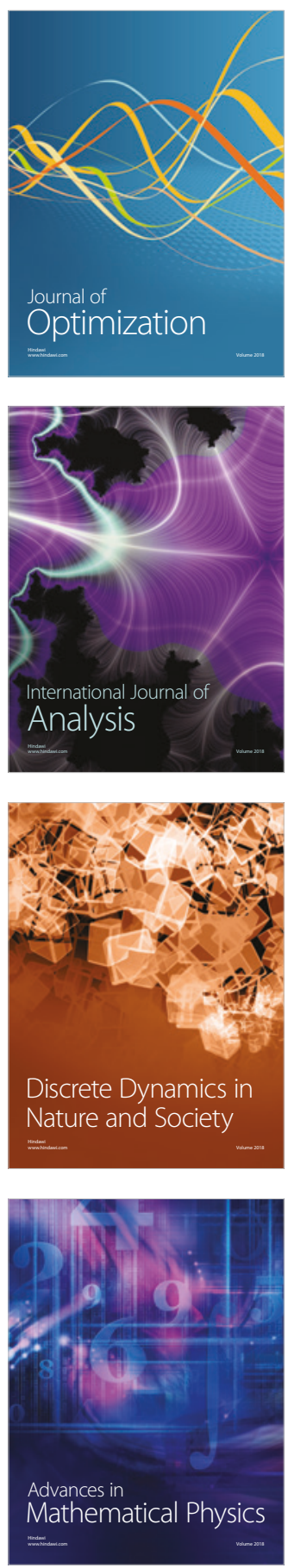